\title{
Magnet Removal and Reinsertion in a Cochlear Implant Recipient Undergoing Brain MRI
}

\author{
Lela Migirov Michael Wolf \\ Department of Otolaryngology and Head and Neck Surgery, Sheba Medical Center Affiliated \\ to Sackler School of Medicine, Tel Aviv University, Tel Hashomer, Israel
}

\section{Key Words}

Magnet removal · Magnetic resonance imaging $\cdot$ Cochlear implant

\begin{abstract}
Background: Recent advances in cochlear implants (CIs) and in magnetic resonance imaging (MRI) techniques have led to increasing numbers of implanted patients who have undergone brain MRIs for various reasons. This paper first describes the surgical procedure for removing the magnet before an MRI and its subsequent reinsertion immediately afterwards in a CI recipient. Method: After the administration of local anesthesia, the magnet was removed by a surgical incision made along the posterior margin of the receiver-stimulator. The flap was elevated and the capsule over the implant was incised. The magnet was removed, maintained under sterile conditions and reinserted immediately after the completion of the 1.5-Tesla MRI. Results: The patient was able to wear her device immediately after surgery. Large CI-associated artifacts were observed on the MRIs irrespective of sequences. The function of the device was not altered by either the MRI or the surgical procedure. Conclusion: The proposed surgical incision that follows the posterior margins of the receiver-stimulator allows the wearing of the device immediately after the surgical procedure. An MRI has limited diagnostic value for lesions located on the implanted side due to unavoidable artifacts, even after the magnet has been removed.

Copyright (C) 2013 S. Karger AG, Basel
\end{abstract}

\section{Introduction}

Recent advances in cochlear implants (CIs) and in magnetic resonance imaging (MRI) techniques have led to increasing numbers of implanted patients who have undergone brain MRIs for various reasons. CI manufacturers have developed devices with removable magnets, 
Migirov et al.: Magnet Removal and Reinsertion in a Cochlear Implant Recipient Undergoing Brain MRI

Fig. 1. Elevation of the flap and incision of the capsule over the implant.

Fig. 2. The magnet has been removed.

Fig. 3. A closed wound after reinsertion of the magnet.
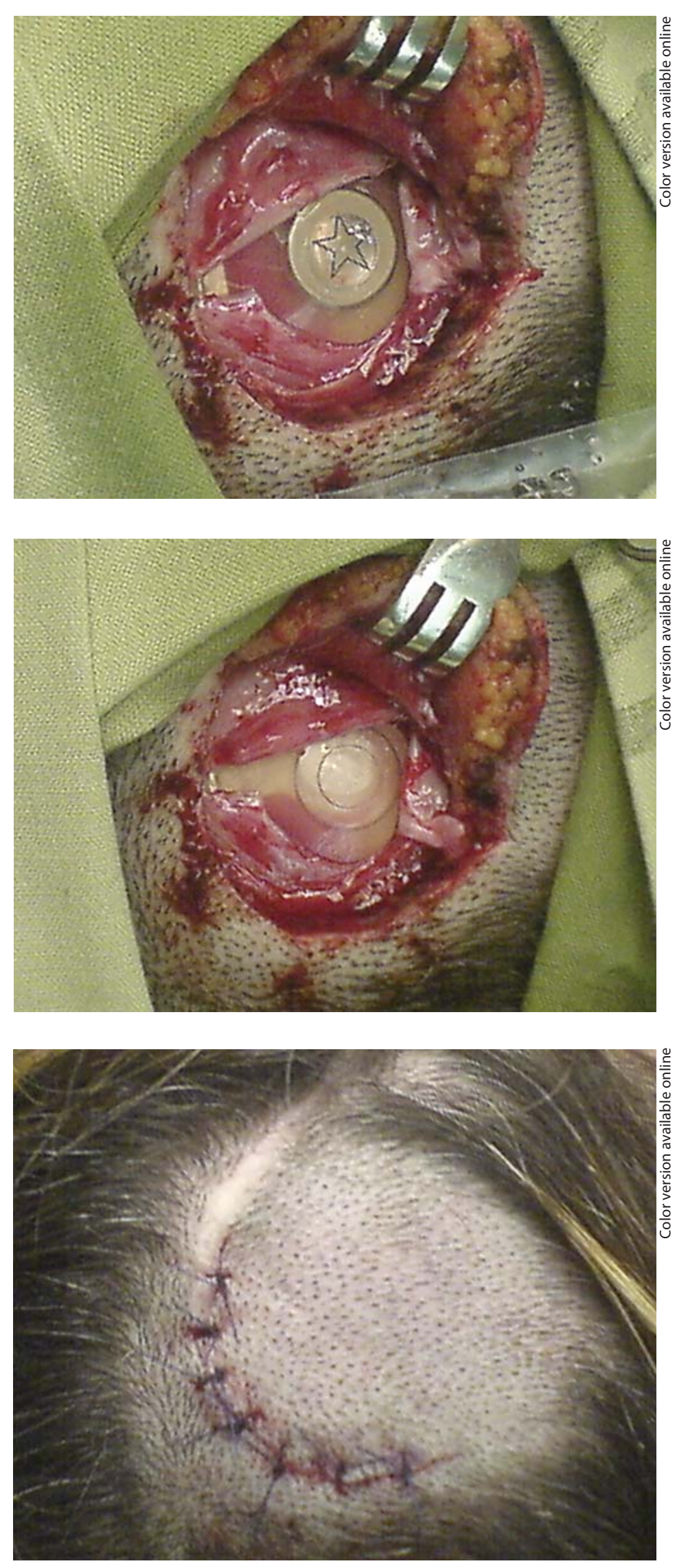
and several articles have addressed the issues related to the MRI compatibility and safety of CIs [1, 2]. Gubbels et al. [1] conducted a cadaveric study and found that performing a 1.5-Tesla MRI on subjects with Cochlear Nucleus 24 implants did not result in any significant demagnetization of the internal magnet, nor was there any displacement of the magnet when an external compression dressing was applied. They concluded that surgical removal of the internal magnet before scanning with the 1.5-Tesla MRI may not be necessary if a compression dressing is applied. However, there are no exact data on the number and outcome of CI recipients who had already undergone MRI studies with and without removing the CI internal magnet, so the controversy over the necessity of its removal remains unresolved $[3,4]$. This is the first paper describing the surgical procedure of magnet removal with subsequent reinsertion in a CI recipient who underwent an MRI.

\section{Case Presentation}

A 33-year-old female with bilateral progressive profound sensorineural hearing loss of unknown origin received a Freedom Contour Nucleus (Cochlear LTD, Sydney, N.S.W., Australia) device in the year 2000. The device provided her with good hearing in the fitted ear. Eight years after her CI, she developed polyneuropathy associated with persistent headache and extreme muscle weakness that required performing an MRI. After the administration of local anesthesia, the magnet was removed by a surgical incision made along the posterior margin of the receiver-stimulator. Care was taken not to perform the incision over the site of magnet, as doing so could interfere with the wearing of the device thereafter. The flap was elevated and the capsule over the implant was incised (fig. 1). The magnet was removed (fig. 2) and maintained under sterile conditions, and the wound was closed with 3 temporary sutures and covered with a sterile pad. The magnet was reinserted immediately after the completion of the 1.5-Tesla MRI, and the wound was closed in two layers (fig. 3). Both the removal and replacement procedures were performed by an experienced CI surgeon (L.M.). The patient was able to wear her device immediately after surgery. A small sterile pad was used to separate the incised skin from the external coil of the device during the first week following surgery. The postoperative course was uneventful. The MRI failed to demonstrate any findings that could explain the patient's neurological condition. Large CI-associated artifacts were observed on the MRIs irrespective of the performed sequences (fig. 4, 5). The function of the device was not altered by the MRI nor by the surgical procedure and the patient is still using her device.

\section{Discussion}

The issue of surgical removal of the internal magnet prior to MRI in CI patients remains controversial. On the one hand, there are large artifacts on the MRI when the magnet is not removed and only a bandage is used during the imaging studies [3]. Crane et al. [3] found that the CI generally produced an artifact on brain MRIs, with a mean maximal anterior-posterior dimension of $6.6 \mathrm{~cm}$ and a lateral dimension of $4.8 \mathrm{~cm}$ around the site of the device. They reported that the contralateral internal auditory canal was visualized in all of their 16 patients, and that the ipsilateral internal auditory canal was at least party visible in all but 1 patient. On the other hand, a surgical procedure for the removal and consequent reinsertion of a magnet can be associated with a risk of local infection at the implant site or even displacement of the magnet when another MRI is performed [4]. Specifically, a failed MRI may require the performance of additional images, this time with the removal of the magnet, and this manipulation can result in CI malfunction. Patients should be informed about the possibility of implant malfunctioning, infection following surgical procedure, magnet displacement during the MRI and reversal of the polarity of the magnet after an MRI [4, 5]. Note that magnet removal is not applicable in implants with ceramic housing, in old models of the Nucleus device with irremovable magnets and in current devices with titanium housing, e.g. from the 


\section{ORL}

\begin{tabular}{l|l}
\hline ORL $2013 ; 75: 1-5$ \\
\hline DOI: $\underline{10.1159 / 000346239}$ & $\begin{array}{l}\text { () } 2013 \text { S. Karger AG, Basel } \\
\text { www.karger.com/orl }\end{array}$ \\
\hline
\end{tabular}

Migirov et al:: Magnet Removal and Reinsertion in a Cochlear Implant Recipient Undergoing Brain MRI

Fig. 4. An axial T2 Flair MRI showing an artifact ipsilateral to the implant side.

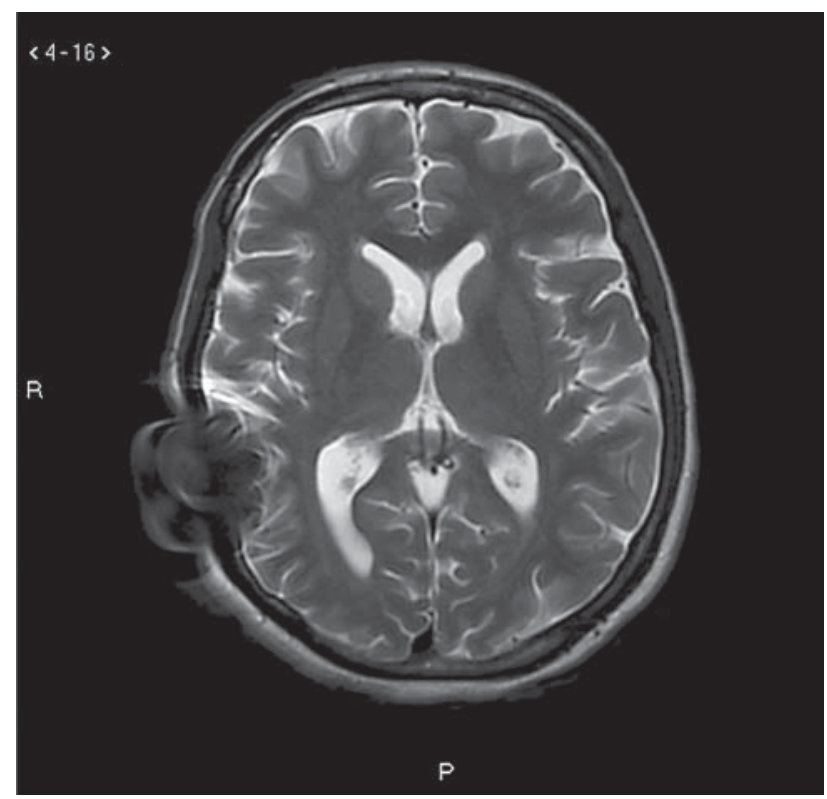

Fig. 5. A coronal 3-dimensional TOF MRI showing a large artifact ipsilateral to the implant side.

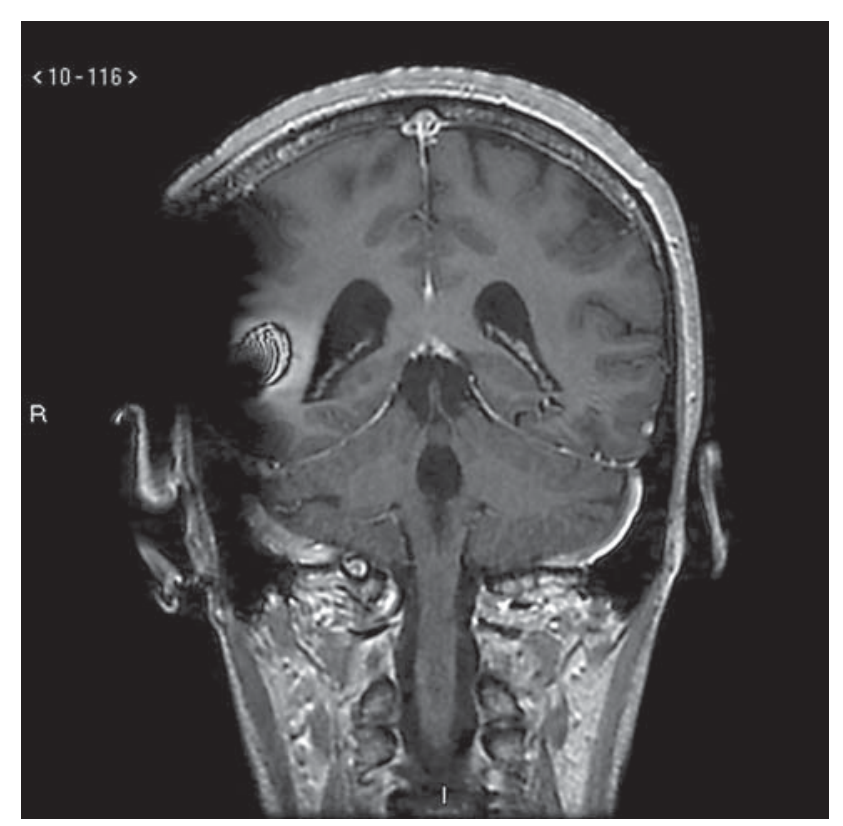

Med-El GmbH, Austria. Thus, it is extremely important to validate the type of the cochlear device before any intervention such as surgery or MRI. For this purpose, we recommend performing a simple X-ray of the skull (lateral view) regardless of the existence of documentation on the kind of the implanted device.

Despite the relative ease of magnet removal and reinsertion, surgeons prefer to perform this procedure only in a small number of implanted patients [3]. This is completely understandable since the surgical procedure is not without risk for the patients as well as for their cochlear devices. The age of $\mathrm{CI}$ candidates has become progressively younger over the years, and young implanted children could require an MRI several times throughout their lifetime. Moreover, when an MRI is planned for a bilaterally implanted child, the surgery for removal 
and reinsertion of both magnets and the performance of the MRI should be done under general anesthesia that can last for more than $2 \mathrm{~h}$. In these specific cases, removal of the magnet for purposes of carrying out MRI studies is, in all probability, essential because otherwise the MRI could very well be rendered nondiagnostic due to the large bilateral artifacts.

\section{Conclusion}

The internal magnet of a CI can be removed and reinserted under local anesthesia, at least in adult recipients. The proposed surgical incision that follows the posterior margins of the receiver-stimulator allows the wearing of the device immediately after the surgical procedure. An MRI has limited diagnostic value for lesions located on the implanted side due to unavoidable artifacts, even after the magnet has been removed.

\section{References}

1 Gubbels SP, McMenomey SO: Safety study of the Cochlear Nucleus 24 device with internal magnet in the 1.5 Tesla magnetic resonance imaging scanner. Laryngoscope 2006;116:865-871.

2 Tognola G, Parazzini M, Sibella F, Paglialonga A, Ravazzani P: Electromagnetic interference and cochlear implants. Ann Ist Super Sanita 2007;43:241-247.

3 Crane BT, Gottschalk B, Kraut M, Aygun N, Niparko JK: Magnetic resonance imaging at $1.5 \mathrm{~T}$ after cochlear implantation. Otol Neurotol 2010;31:1215-1220.

- 4 Deneuve S, Loundon N, Leboulanger N, Rouillon I, Garabedian EN: Cochlear implant magnet displacement during magnetic resonance imaging. Otol Neurotol 2008;29:789-790.

5 Jeon JH, Bae MR, Chang JW, Choi JY: Reversing the polarity of a cochlear implant magnet after magnetic resonance imaging. Auris Nasus Larynx 2012;39:415-417. 